ORIGINAL PAPER

Kim A. Margolin · P. Y. Liu · Joseph M. Unger

William S. Fletcher - Lawrence E. Flaherty - Walter J. Urba

Evan M. Hersh · Laura E. Hutchins · Jeffrey A. Sosman

John W. Smith · Geoffrey R. Weiss · Vernon K. Sondak

\title{
Phase II trial of biochemotherapy with interferon $\alpha$, dacarbazine, cisplatin and tamoxifen in metastatic melanoma: a Southwest Oncology Group trial
}

Received: 11 August 1998 / Accepted: 21 December 1998

Abstract The therapeutic benefit of adding interferon $\alpha$ (IFN $\alpha$ ) to established single-agent and combination chemotherapy regimens for the treatment of metastatic melanoma has not been proven. We designed the present study to estimate the response rate of IFN $\alpha$, dacarbazine, cisplatin and tamoxifen in patients who had not been treated with systemic therapy for advanced disease. Using a schedule similar to that which had

This investigation was supported in part by the following PHS Cooperative Agreement grant numbers awarded by the National Cancer Institute, DHHS: CA38926, CA32102, CA46368, CA46113, CA42028, CA45377, CA13612, CA37981, CA46282, CA27057, CA22433.

K.A. Margolin $(\square)$

Department of Medical Oncology,

City of Hope National Medical Center,

1500 East Duarte Road, Duarte, CA 91010-3000 USA

Tel.: + 1 626-359 8111 (ext 2307)

Fax: + 1 626-3018898

P.Y. Liu · J.M. Unger

Southwest Oncology Group Statistical Center, Seattle, WA, USA

W.S. Fletcher

Oregon Health Sciences University, Portland, OR

L.E. Flaherty

Wayne State University Medical Center, Detroit, MI

W.J. Urba · J.W. Smith, II

Columbia River CCOP, Portland, OR

E.M. Hersh

University of Arizona Cancer Center, Tucson, AZ

L.E. Hutchins

University of Arkansas for Medical Science, Little Rock, AR

J.A. Sosman

University of Illinois at Chicago, IL

G.R. Weiss

University of Texas Health Science Center at San Antonio, TX

V.K. Sondak

University of Michigan Medical Center, Ann Arbor, MI previously been shown to favor IFN $\alpha$ plus dacarbazine over dacarbazine alone, we treated patients with an "induction" regimen of IFN $\alpha, 15 \mathrm{mU} \mathrm{m}^{-2}$ day $^{-1}$ intravenously 5 days/week for 3 weeks. Following induction, schedules of IFNa, $5 \mathrm{mU} \mathrm{m}^{-2}$ day $^{-1}$ subcutaneously three times a week, and tamoxifen, $10 \mathrm{mg}$ orally twice a day, were begun. Dacarbazine, $250 \mathrm{mg} \mathrm{m}^{-2} \mathrm{day}^{-1}$ and cisplatin $33 \mathrm{mg} \mathrm{m}^{-2}$ day $^{-1}$ for 3 consecutive days were repeated every 4 weeks, and subcutaneous IFN $\alpha$ and oral tamoxifen were continued until the discontinuation of chemotherapy. We treated 25 patients (18 men and 7 women, median age 52 years) and observed only 1 objective response (response rate 4\%, 95\% confidence interval $0.1 \%-20 \%)$. The toxicities of the regimen consisted of moderate myelosuppression and constitutional side-effects. On the basis of the low antitumor activity of this regimen, we do not recommend it for further study or for use as standard therapy of metastatic melanoma.

Key words Melanoma $\cdot$ Interferon $\cdot$ Biochemotherapy

Abbreviation IFN interferon

\section{Introduction}

The addition of biological response modifiers such as interferon $\alpha$ (IFN $\alpha$ ) to selected chemotherapeutic agents has additive or even synergistic effects in preclinical models of human solid tumors (Wadler and Schwartz 1990). The therapeutic benefit of adding biological response modulators to chemotherapy for the treatment of patients with advanced melanoma has not been proven, although the results of small randomized and nonrandomized studies have suggested the superiority of aggressive biochemotherapy combinations over either component alone (Legha et al. 1998; Richards et al. 1992; Atkins et al. 1994; Khayat et al. 1993; Keilholz et al. 1997). 
The results of phase II studies showed encouraging activity for combinations of IFN $\alpha$ and dacarbazine [5(3,3-dimethyl-1-triazenyl)-1 $H$-imidazole-4-carboxamide, DTIC] (Hersey et al. 1991; Bajetta et al. 1990), but randomized studies did not provide a definitive conclusion as to the benefit of adding IFN $\alpha$ to dacarbazine (Thomson et al. 1993; Bajetta et al. 1994). However, a subsequent phase III trial of intravenous IFN $\alpha$, administered daily 5 days/week for 3 weeks, followed by dacarbazine and thrice-weekly subcutaneous IFN $\alpha$ showed a significantly improved objective response rate of $53 \%$ with the combination, compared to only $20 \%$ with dacarbazine alone. Complete responses were seen only in patients assigned to the combination regimen. The rationale for the dose and schedule of IFN $\alpha$ was to achieve exposure to high peak levels followed by continuous exposure to the biological agent while safely combining it with dacarbazine at full doses (Falkson et al. 1991).

We designed the present study to evaluate the feasibility of combining IFN $\alpha$ with dacarbazine, cisplatin and tamoxifen, using the same sequence of IFN $\alpha$ and chemotherapy as used in that trial. The chemotherapy combination was based on a series of Southwest Oncology Group (SWOG) regimens evaluating dacarbazine and cisplatin alone (Fletcher et al. 1993) or with tamoxifen (Flaherty et al. 1996), a modulator that has been considered critical for the optimal antitumor activity of one or more of the active drugs (McClay et al. 1989). Although nitrosoureas are often incorporated into combination chemotherapy for advanced melano$\mathrm{ma}$, their precise role has never been firmly established in prospective trials, and we felt that the prolonged myelosuppression associated with $N, N^{\prime}$-bis(2-chloroethyl)$N$-nitrosourea, particularly the risk of cumulative thrombocytopenia, would make it difficult to combine with continuous IFN $\alpha$. We sought to identify an active and tolerable regimen that could subsequently form the basis of a randomized study to determine the benefit of combining IFN $\alpha$ with chemotherapy in the treatment of advanced melanoma or in the adjuvant setting.

\section{Patients and methods}

Patient eligibility

The eligibility criteria for this trial included the following clinical parameters: histological diagnosis of melanoma with measurable metastatic disease, a SWOG performance status of 1 or lower, no history of seizure disorder or central nervous system metastasis (brain computed tomographic or magnetic resonance imaging required), no prior therapy with any of the agents in the regimen (except adjuvant IFN $\alpha$ if completed at least 12 months prior to relapse), and no active infection or anticipated need for glucocorticoid therapy. Patients with a history of serious ventricular arrhythmia, congestive heart failure, poorly controlled angina pectoris, or other severe cardiovascular disease were excluded. At least 28 days had to have elapsed since prior radiation therapy, and the patient had to have recovered from any prior surgery. Patients were allowed one prior chemotherapy for advanced disease. Laboratory parameters were as follows: serum bilirubin at or within the institutional upper limit of normal, alanine aminotransferase at or within $2.5 \times$ the institutional upper limits of normal $(\leq 5 \times$ the institutional upper limits of normal if the liver was involved with tumor), granulocytes at least $1800 / \mu \mathrm{l}$, platelet count at least $150000 / \mu \mathrm{l}$, and hemoglobin at least $10 \mathrm{~g} / \mathrm{dl}$; serum creatinine had to be within normal limits and the estimated or measured creatinine clearance at least $60 \mathrm{ml} / \mathrm{min}$. Tumor measurements were performed within 28 days of study registration. All patients provided their voluntary, written informed consent to participate in the study, which was approved by the institutional review boards of each of the participating institutions.

\section{Treatment}

Treatment was administered in the inpatient or outpatient units of the participating institutions to assure adequate hydration and management of the volume and electrolyte status of the patients. Antiemetic therapies were at the treating physician's discretion, but corticosteroids were excluded. Treatment was initiated with IFNa alone, $15 \mathrm{mU} \mathrm{m}^{-2} \mathrm{day}^{-1}$, intravenously (i.v.) 5 days/week for 3 weeks; this was followed by chemotherapy consisting of dacarbazine, $250 \mathrm{mg} \mathrm{m}^{-2}$ day $^{-1}$ i.v., and cisplatin, $33 \mathrm{mg} \mathrm{m}^{-2}$ day $^{-1}$ i.v., on days $1-3$. Tamoxifen, $10 \mathrm{mg}$ orally, twice daily, was begun with chemotherapy and continued throughout protocol treatment. IFN $\alpha$ was resumed at $5 \mathrm{mU} / \mathrm{m}^{2}$ subcutaneously (s.c.), three times weekly, on the first day of chemotherapy and continued throughout protocol treatment.

Chemotherapy cycles were repeated every 4 weeks in responding and stable patients who continued to meet parameters for treatment (see below). The maximum duration of therapy was 1 year. During protocol therapy, patients were required to undergo the following clinical and laboratory examinations: a weekly complete blood count; electrolytes, creatinine, urinalysis and liver function tests; biweekly physical examination and toxicity assessments (using the National Cancer Institute Toxicity grading criteria). The creatinine clearance was measured or calculated prior to each chemotherapy cycle.

Guidelines for dose adjustments of the therapeutic agents and the use of colony-stimulating factors after the first cycle of therapy were as follows: IFN $\alpha$ could be continued despite grade 1 toxicity; for IFN $\alpha$-related toxicities of grade 2 or more, IFN $\alpha$ was to be held until all toxicities resolved to grade 1 or less, followed by resumption of therapy at $50 \%$ of the prior dose. Dose re-escalations were not permitted. If toxicity of grade 2 or more recurred at the decreased dose, the patient was to be taken off IFN $\alpha$ and could be continued on chemotherapy. Cisplatin dose adjustments were provided for renal impairment and peripheral neuropathy. Dacarbazine doses were adjusted for nadir and treatment-day cytopenias, and treatment could be delayed up to 2 weeks to allow sufficient recovery for full dose administration. Granulocyte-colony-stimulating factor (G-CSF) was to be added in the event of fever with neutrophils at $1000 / \mu l$ or less, in which case the dacarbazine dose for the following cycle was to be decreased by $25 \%$ and $5 \mu \mathrm{g} /$ kg G-CSF used prophylactically on days 4-9 (or until the neutrophil count was at least $1500 / \mu 1$ for 3 consecutive days). Guidelines were also provided for management of thromboembolic complications from tamoxifen, but no such episode was observed in this study.

\section{Evaluation of response}

The definitions of response were as previously described for Southwest Oncology Group studies (Green and Weiss 1992). Scans for tumor assessment were to be repeated after every two cycles. For confirmation of the response status, a repeat tumor assessment was required 4 weeks following the first achievement of response. The maximum duration of therapy was 1 year. Patients achieving complete response were to receive two additional treatment cycles following confirmation of complete response. Patients who showed evidence of disease progression after the initial IFN $\alpha$ but prior to 
chemotherapy were allowed to remain on-study as long as they continued to meet the eligibility criteria.

\section{Statistical design}

The primary objective of this pilot study was to assess the antitumor activity of the regimen, which would be considered worthy of further study if the true response rate was $35 \%$ or more and not of interest if it was below $20 \%$. The study was designed to accrue 60 patients in a single stage. Out of 60 patients, 18 responses would represent evidence of antitumor activity worthy of further pursuit and would reject a response rate of $20 \%$ or less at a one-sided 0.05 level of significance (or better) and provide a power of $83 \%$ or more to detect a true response rate of at least 35\% (Green and Dahlberg 1992). The second objective was to evaluate the toxicity and tolerability of the regimen. It would be considered unacceptable for standard use or for inclusion in a phase III trial if either (a) more than $60 \%$ of patients experienced grade 3 or worse nonhematological toxicity or thrombocytopenia during the i.v. IFN $\alpha$ treatment, or (b) more than $60 \%$ of the patients required the discontinuation of i.v. IFN $\alpha$ during the first treatment cycle.

\section{Results}

\section{Patient characteristics}

Between August 1994 and November 1996, 26 patients were accrued at nine Southwest Oncology Group Institutions. One patient was found to be ineligible because of missing study documentation. Patient characteristics are listed in Table 1 . Of the 25 eligible patients, there were 18 men and 7 women, and the median age was 52 years (range 17-75 years). Twenty-three patients were Caucasian, 1 was black and 1 Hispanic. Thirteen patients had performance status 0 and 12 had performance status 1 . Only 2 patients had metastatic disease limited

Table 1 Patient characteristics $(n=25)$

\begin{tabular}{lcc}
\hline Characteristic & No. & $\%$ \\
\hline Age (years), median (range) & $52(17-75)$ & \\
Sex & 18 & 72 \\
$\quad$ Male & 7 & 28 \\
Female & & \\
Race & 23 & 92 \\
$\quad$ White & 1 & 4 \\
Black & 1 & 4 \\
Hispanic & & \\
Performance status & 13 & 52 \\
0 & 12 & 48 \\
1 & & \\
Sites of active disease & 2 & 92 \\
Skin and/or nodes only & 23 & 24 \\
Visceral & 6 & 72 \\
Liver & 18 & 12 \\
Lung & 3 & \\
Bone & & 44 \\
Number of metastatic sites & 11 & 32 \\
1 & 8 & 24 \\
2 & 6 & \\
$\geq 3$ & &
\end{tabular}

to skin and/or soft tissue/lymph nodes, and the rest had visceral involvement [liver, 6 (24\%); lung, 18 (72\%); bone, $3(12 \%)$ ]. No patient had received previous chemotherapy or biological response modifiers.

Although a formal two-stage accrual design was not part of the original statistical plan, we had the opportunity to analyze the data after the first 26 patients, when a national shortage of dacarbazine occurred. Although the toxicity level of this regimen did not exceed that which was allowable by the criteria detailed above, there had been only a single confirmed objective response among the first 26 patients ( $95 \%$ confidence interval $\leq 20 \%)$, so we elected to close the study to further accrual.

\section{Treatment administered}

Although organ toxicities requiring dose adjustment were rare (Table 2), administration of IFN $\alpha$ was often limited by constitutional side-effects (fever, myalgias, severe fatigue, nausea, and anorexia). Seventeen patients $(68 \%)$ received all of the i.v. IFN $\alpha$ planned for cycle 1 , but only $6(24 \%)$ received $100 \%$ of the planned i.v. and s.c. IFN $\alpha ; 11$ patients $(44 \%)$ completed all of the i.v. IFN $\alpha$ but required dose reductions of s.c. therapy, and 3 $(12 \%)$ discontinued the i.v. phase early but completed the planned s.c. dosing; 5 patients $(20 \%)$ required dose adjustments of both phases of therapy.

The median duration of protocol therapy was 3 months, corresponding to "induction" plus two cycles of combination therapy, and the predominant reason for going off study was disease progression (in 15 patients, $60 \%$ ). The other reasons for discontinuation of protocol therapy were toxicity of therapy (4 patients) or patient refusal ( 3 patients); 3 patients were taken off study for reasons related to physician judgment or insurance limitations without progressive disease or toxicity requiring removal from study.

\section{Toxicities of treatment}

The grade 3 and 4 non-constitutional toxicities of the regimen are summarized in Table 2 and are listed separately for the initial 3-week period of intravenous IFN $\alpha$ and the subsequent combined chemotherapy and sub-

Table 2 Toxicities of therapy $(n=25)$. IFN interferon

\begin{tabular}{llll}
\hline Therapy & Toxicity & Grade 3 & Grade 4 \\
\hline Intravenous & Anemia & 1 & \\
IFN $\alpha$ & Granulocytopenia & 2 & \\
& Thrombocytopenia & & 1 \\
& Bilirubin elevation & 1 & \\
Transaminase elevation & 2 & \\
phemotherapy & Anemia & 2 & \\
plus s.c. IFN $\alpha$ & Granulocytopenia & 7 & 4 \\
& Thrombocytopenia & 3 & 1 \\
& Bilirubin elevation & 1 & \\
\hline
\end{tabular}


cutaneous IFNa "biochemotherapy." The spectrum of toxicities was in accordance with those previously described for high-dose intravenous IFNa. The toxicities associated with the full multi-agent regimen were neither unexpected nor excessively severe, consisting mainly of nausea, mild to moderate neutropenia and thrombocytopenia, which were managed by dose adjustments and, if indicated, the institution of G-CSF according to protocol requirements.

\section{Tumor response}

There was one partial response (duration 7 months) in a man with lung metastases, among the 25 eligible patients (objective response rate $4 \%, 95 \%$ confidence interval $0.1 \%-20 \%$ ). The study was closed temporarily after 26 patients had been accrued ( 25 eligible) because of a national shortage of dacarbazine. Analysis of these first 25 eligible patients revealed that there had been only 1 objective response, providing an exact $95 \%$ confidence interval of $0.1 \%-20 \%$ for the response rate. This result was consistent with the null hypothesis of a $20 \%$ or lower response rate, while the hypothesis of a response rate of at least $35 \%$ was rejected with a $P$ value of 0.0003 . Therefore, the study was permanently closed.

The median survival of the entire group was 10 months $(95 \%$ confidence interval $7-18$ months).

\section{Discussion}

Current clinical investigations in melanoma therapy are focused in two main areas: the discovery of more active chemotherapeutic and/or biological molecules, and the identification of the most active regimen for standard therapy, based on currently available agents. The study reported here was an attempt to combine a chemohormonal combination that had previously been studied by the Southwest Oncology Group (Flaherty et al. 1996), with an aggressive dose and schedule of IFN $\alpha$, employing a sequence that had shown promising enhancement of antitumor activity when combined with single-agent dacarbazine. (Falkson et al. 1991) Since the results of cooperative group phase II and III studies have almost invariably shown lower objective response rates than smaller, usually single-institution studies of identical regimens (Flaherty et al. 1997), we chose to pilot the present regimen in the cooperative group setting prior to incorporating this regimen into a phase III biochemotherapy versus chemotherapy study. Participation in this trial was limited to a select group of SWOG institutions and investigators who had demonstrated capability for administering aggressive biochemotherapy combinations to patients with advanced melanoma.

The patients enrolled in this study had a significant tumor burden, as evidenced by the high proportion of patients with visceral (particularly hepatic) involvement and the number of patients with a compromised performance status. All of these factors have been associated with a lower response to systemic therapy in cooperative group melanoma trials (Flaherty et al. 1997). This factor may account, in part, for the low overall response rate in our study, despite an aggressive regimen delivered by a small group of experienced clinicians.

The apparent lack of enhanced antitumor activity resulting from the addition of IFN $\alpha$ to chemotherapy on this dose and schedule is consistent with the recently reported results of the Eastern Cooperative Oncology Group (ECOG), in which the addition of IFN $\alpha$ in the same dose and schedule as dacarbazine, with or without tamoxifen, did not enhance the objective response rate or the survival of patients with metastatic melanoma (Falkson et al. 1998). The results of our study, initiated before the preliminary ECOG analysis (Falkson et al. 1996), are compatible with the findings of this randomized trial.

It is possible that the schedule of biochemotherapy that we used for this trial led to antagonistic rather than additive or synergistic interactions with one or both of the chemotherapeutic agents. The dependence of optimal biochemotherapy interactions on the schedule is well-described (Welander 1987) but has not been addressed adequately in human cancer therapy trials. We based our schedule and doses of IFN $\alpha$ on that which had shown superiority to chemotherapy alone in the prior randomized trial (see above). Furthermore, in the adjuvant setting, an "induction" phase consisting of intensive intravenous IFN $\alpha$ followed by a relatively aggressive regimen of thrice-weekly subcutaneous IFN $\alpha$, appeared to be essential for the activity of this agent in prolongation of disease-free and overall survival (Kirkwood et al. 1996), while regimens consisting of lower doses and lacking an induction phase have been inactive (reviewed in Sondak and Margolin 1999).

The results of our study do not exclude a beneficial role for optimally scheduled addition of IFN $\alpha$ in combinations for advanced melanoma. This question is currently being tested by the SWOG/ECOG Intergroup in a randomized phase III trial of combination chemotherapy [the cisplatin, vinblastine, dacarbazine (CVD) regimen of Legha et al.] with or without IFN $\alpha$ and infusional interleukin-2 (IL-2). The chemotherapy combination chosen for this study, while never proven to be better than dacarbazine alone in a completed phase III trial, has been well-tolerated and associated with objective response rates in the range of $40 \%$ in large single-institution studies (Legha et al. 1989). It has also been combined successfully with IFN $\alpha$ and IL2 in a regimen reported to induce durable complete responses in $8 \%-10 \%$ of patients (Legha et al. 1998). The goal of this new study is to compare not only the objective response rates but also the percentage of patients achieving durable complete responses, an outcome that, to date, has been reported only in trials 
of aggressive biochemotherapy. The results of this trial as well as ongoing innovations in the field of melanoma immunotherapy will define future approaches to the therapy of advanced disease and the adjuvant therapy of high-risk primary melanoma.

\section{References}

Atkins MB, O'Boyle KR, Sosman JA, Weiss GR, Margolin KA, Ernest ML, Kappler K, Mier JW, Sparano JA, Fisher RI, Eckardt JR, Pereira C, Aronson FR (1994) Multiinstitutional phase II trial of intensive combination chemoimmunotherapy for metastatic melanoma. J Clin Oncol 12:1553-1560

Bajetta E, Negretti E, Giannotti B, Brogelli L, Brunelli I, Sertoli MR, Bernengo MG, Sofra MC, Maifredi G, Zumiani G, Comella G, Buzzoni R, Di Leo A, Criscuolo D, Massimini G, Cascinelli N (1990) Phase II study of interferon $\alpha$-2a and dacarbazine in advanced melanoma. Am $\mathbf{J}$ Clin Oncol (CCT) 13:405-409

Bajetta E, Di Leo A, Zampino MG, Sertoli MR, Comella G, Barduagni M, Giannotti B, Queirolo P, Tribbia G, Bernengo MG, Menichetti ET, Palmeri S, Russo A, Cristofolinio M, Erbazzi A, Fowst C, Criscuolo D, Bufalino R, Zilembo N, Cascinelli N (1994) Multicenter randomized trial of dacarbazine alone or in combination with two different doses and schedules of interferon alfa-2a in the treatment of advanced melanoma. J Clin Oncol 12:806-811

Falkson CI, Falkson G, Falkson HC (1991) Improved results with the addition of interferon alfa- $2 b$ to dacarbazine in the treatment of patients with metastatic malignant melanoma. J Clin Oncol 9:1403-1408

Falkson CI, Ibrahim J, Kirkwood JM, et al. (1996) A randomized phase III trial of dacarbazine (DTIC) versus DTIC + interferon $\alpha$-2b (IFN) versus DTIC + tamoxifen (TMX) versus DTIC + IFN + TMX in metastatic malignant melanoma: an ECOG trial. Proc Am Soc Clin Oncol 15, 1350:435

Falkson CI, Ibrahim J, Kirkwood, JM, et al (1998) Phase III Trial of Dacarbazine versus Dacarbazine with Interferon $\alpha-2 b$ versus Dacarbazine with Tamoxifen versus Dacarbazine with Interferon $\alpha 2 \mathrm{~b}$ and Tamoxifen in Patients with Metastatic Malignant Melanoma: An Eastern Cooperative Oncology Group Study J Clin Oncol 16: 1743-1751

Flaherty LE, Liu PY, Mitchell MS, et al. (1996) The addition of tamoxifen to dacarbazine and cisplatin in metastatic malignant melanoma. Am J Clin Oncol 19:108-113

Flaherty L, Liu PY, Unger J, Sondak V (1997) Comparison of patient characteristics and outcome between a single institution phase II trial and a cooperative-group phase II trial with identical eligibility in metastatic melanoma. Am J Clin Oncol (CC) 20:600-604

Fletcher WS, Daniels SD, Sondak VK, et al. (1993) Evaluation of cisplatin and DTIC in inoperable stage III and IV melanoma. Am J Clin Oncol 16:359-362
Green SJ, Dahlberg S (1992) Planned versus attained design in phase II clinical trials. Stat Med 11:853-862

Green SJ, Weiss GR (1992) Southwest Oncology Group standard response criteria, endpoint definitions and toxicity criteria. Invest New Drugs 10:239-253

Hersey P, McLeod GRC, Thomson DB (1991) Treatment of advanced malignant melanoma with recombinant interferon alfa2a in combination with DTIC: Long-term follow-up of two phase II studies. Br J Haematol 79:60-66

Keilholz U, Goey SH, Punt C JA, Proebstle TM, Salzmann R, Scheibenbogen C, Schadendorf D, Liénard D, Enk A, Dummer R, Hantich B, Geueke AM, Eggermont MM (1997) Interfon alfa-2a and interleukin-2 with or without cisplatin in metastatic melanoma: a randomized trial of the European Organization for Research and Treatment of Cancer Melanoma Cooperative Group. J Clin Oncol 15:2579-2588

Khayat D, Borel C, Tourani JM, et al. (1993) Sequential chemoimmunotherapy with cisplatin, interleukin-2, and interferon alfa-2a for metastatic melanoma. J Clin Oncol 11:2173-2180

Kirkwood JM, Hunt-Strawderman M, Ernstoff MS, Smith TJ, Borden EC, Blum RH (1996) Interferon alfa-2b adjuvant therapy of high-risk resected cutaneous melanoma: the Eastern Cooperative Oncology Group trial EST 1684. J Clin Oncol 14:7-17

Legha SS, Ring S, Papadopoulos N, et al. (1989) A prospective evaluation of a triple-drug regimen containing cisplatin, vinblastine, and dacarbazine (CVD) for metastatic melanoma. Cancer 64:2024-2029

Legha SS, Ring S, Eton O, et al. (1998) Development of a biochemotherapy regimen with concurrent administration of cisplatin, vinblastine, dacarbazine, interferon alfa, and interleukin-2 for patients with metastatic melanoma. J Clin Oncol 16:1752-1759

McClay EF, Mastrangelo MJ, Sprandio JD, Bellet RE, Berd D (1989) The importance of tamoxifen to a cisplatin-containing regimen in the treatment of metastatic melanoma. Cancer 63:1292-1295

Richards JM, Mehta N, Ramming K, Skosey P (1992) Sequential chemoimmuno-therapy in the treatment of metastatic melanoma. J Clin Oncol 10:1338-1343

Sondak VK, Margolin KA (1999) Melanoma and other skin cancers. In: Pazdur R, et al. (eds) Cancer Management: A Multidisciplinary Approach 3rd Ed, PRR, Huntington, NY, In Press

Thomson DB, Adena M, McLeod GR, Hersey P, Gill PG, Coates AS, Olver IN, Kefford RF, Lowenthal RM, Beadle GF, et al. (1993) Interferon $\alpha$-2a does not improve response or survival when combined with dacarbazine (DTIC) in metastatic melanoma: results of a multi-institutional Australian randomized trial. Melanoma Res 3:133-138

Wadler S, Schwartz EL (1990) Antineoplastic activity of the combination of interferon and cytotoxic agents against experimental and human malignancies: a review. Cancer Res 50:3473-3486

Welander CE (1987) Overview of Preclinical and Clinical Studies of Interferon Alfa-2b in combination with Cytoxic Drugs. Invest New Drugs 5:S47-S59 